\title{
Characteristics and outcome of laryngeal squamous cell carcinoma in young adults
}

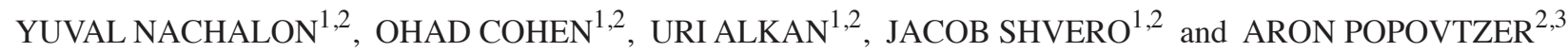 \\ ${ }^{1}$ Department of Otorhinolaryngology-Head and Neck Surgery, Rabin Medical Center, Beilinson Hospital, Petah Tikva 4941492; \\ ${ }^{2}$ Department of Clinical Otolaryngology, Sackler Faculty of Medicine, Tel Aviv University, Tel Aviv 6997801; \\ ${ }^{3}$ Davidoff Cancer Center, Rabin Medical Center, Beilinson Hospital, Petah Tikva 4941492, Israel
}

Received June 10, 2016; Accepted September 27, 2016

DOI: $10.3892 / \mathrm{ol} .2016 .5528$

\begin{abstract}
Laryngeal carcinoma rarely occurs in the young adult population. Therefore, the optimal treatment for this age group is unclear, specifically regarding organ preservation treatment. In order to assess the distinct characteristics of laryngeal squamous cell carcinoma (SCC) in young adults and describe the effect of treatment on survival, a retrospective chart review of all patients aged $<40$ years, who were treated in a tertiary referral center for laryngeal SCC between January 1960 and December 2013, was performed. Patients who were treated prior to and following the Veterans study, representing an arbitrary point which started the organ preservation era, were compared. A total of 29 patients (male:female ratio, 2.6:1) were identified. The mean age at diagnosis was $35 \pm 5$ years and 17 patients $(59 \%)$ were smokers. In total, $12(41 \%)$ of patients were stage I, 4 (14\%) were stage II, 8 (28\%) were stage III and $5(17 \%)$ were stage IV. Glottic tumors were present in $20(69 \%)$ of patients and supraglottic tumors in $6(21 \%)$; the site of tumor origin could not be determined in $3(10 \%)$ of patients. Surgery was performed in $11(38 \%)$ of patients, radiation in $21(72 \%)$ and chemotherapy in $5(17 \%)$. A comparison between patients treated prior to and following the Veterans study demonstrated a 2-year higher laryngectomy-free survival rate of 53\% and $78 \%$, respectively $(\mathrm{P}=0.299)$. The 2 -year disease-free survival rate was $93 \%$ for patients who were treated prior to the Veterans study and $71 \%$ for patients who were treated after $(\mathrm{P}=0.001)$, with no significant change in overall survival $(\mathrm{P}=0.413)$. The results suggest that the characteristics and behavior of laryngeal carcinoma in young adults is similar to older adults. Higher rates of 2-year laryngectomy-free survival were noted in patients treated following the organ preservation era with no
\end{abstract}

Correspondence to: Dr Yuval Nachalon, Department of Otolaryngology-Head and Neck Surgery, Rabin Medical Center, Beilinson Hospital, 39 Jabutinski Street, Petah Tikva 4941492, Israel E-mail: yuval.nachalon@gmail.com

Key words: laryngectomy, survival, larynx, squamous cell carcinoma, young significant difference in survival compared with patients who were treated before.

\section{Introduction}

Laryngeal carcinoma is the second most prevalent malignancy of the upper aerodigestive tract, accounting for 3,620 mortalities per year in the United States. The estimated incidence in 2016 was 13,430 new cases in the US (1). Smoking and alcohol ingestion are known risk factors with a synergistic effect (2). A dose-dependent effect of cigarette smoking has been observed, and the risk for cancer remains elevated even several years after smoking cessation (3).

Less than $10 \%$ of patients with laryngeal cancer are younger than 40 years of age. The association of classic risk factors for squamous cell carcinoma (SCC) of the larynx in young patients is less prominent compared with older patients $(4,5)$. This may imply a different disease than classic larynx cancer with a unique pathophysiology. Other risk factors, including human papilloma virus and laryngopharyngeal reflux, remain under investigation and their link to laryngeal cancer is controversial (6-9).

Up until the 1990's, the primary treatment for laryngeal SCC was surgery, which included various types, such as total or partial laryngectomy. The Veterans Affairs laryngeal cancer study (VA) changed this paradigm and the pendulum shifted towards treatments that preserve the larynx, including radiotherapy (10). However, the optimal treatment of laryngeal SCC in young patients remains unclear.

The aim of the present study was to assess the distinct characteristics of laryngeal SCC in patients less than 40 years of age and the effect of the organ preservation era on survival.

\section{Materials and methods}

Design and setting. The present study was a retrospective chart review based in the Rabin Medical Center, a tertiary university-affiliated center in Petah Tikva, Israel.

Participants. All patients were $<40$ years of age, and had been treated for SCC of the larynx between January 1960 and December 2013. Patients who were treated prior to the VA were compared with those treated after. The VA was 
selected arbitrarily as a reference point that initiated the organ preservation era (OPE). Parameters included patient age and gender, presenting symptoms, histological diagnosis, treatment modalities, recurrence and survival time. The study was approved by the Rabin Medica Center Institutional Ethics Committee.

Statistical analysis. Data were analyzed using SPSS v22.0 (IBM SPSS, Armonk, NY, USA). Survival time was calculated using the Kaplan-Meier product limit estimate method. Variables were compared between groups using T-scores and the $\chi^{2}$ test. $\mathrm{P}<0.05$ was considered to indicate a statistically significant difference.

\section{Results}

Patient demographics and clinical data. Patient demographic and clinical data are presented in Table I. A total of 29 patients (male:female ratio, 2.6:1) were identified and the mean age at diagnosis was $35 \pm 5$ years. In total, 17 patients were smokers and 1 reported alcohol abuse. The mean number of packs per year was $32 \pm 14.4$ (range, 10-60) and was identified to have no significant effect on survival $(\mathrm{P}=0.326)$. Hoarseness was the most common presenting symptom and only a minority of patients reported dysphagia, odynophagia or dyspnea. The glottis was the most common site of disease (69\%), while supraglottic tumors were noted in $21 \%$ of patients. The site of origin could not be determined in $10 \%$ of patients. A total of 5 patients $(17 \%)$ presented with stage IV disease and $8(28 \%)$ presented with stage III. The rest of the patients presented at an early stage. Surgery was performed in 11 patients, with 7 undergoing an upfront total laryngectomy and 2 undergoing supraglottic laryngectomy and hemilaryngectomy. A total of 21 patients were treated with radiotherapy and 5 patients with chemotherapy; 2 patients were given induction chemotherapy. Tumor grade was reported in 14 patients, $50 \%$ had G2 histology. The rest of the patients presented with well- or poorly-differentiated tumors.

Patient outcome and survival. The mean duration of follow-up was 18.7 \pm 18.8 years (range, 1-57 years). Overall survival (OS) time for the entire cohort was $30.8 \pm 4.6$ years. Stage IV and III patients had an OS time of $5.4 \pm 1.4$ and $10.5 \pm 2.2$ years, respectively, with a significantly poorer outcome than patients with earlier stages $(\mathrm{P}=0.043)$ (Fig. 1). The 2- and 5-year survival rates for the entire cohort were 86 and $79 \%$ respectively. Disease site or smoking history was not observed to affect survival. A total of 15 patients succumbed during follow-up. Of the 12 patients in whom disease recurred, local recurrence was most common.

Effect of organ preservation treatment on patients with laryngeal SCC. To assess the effect of organ preservation treatment in young patients, patients who were treated prior to the VA were compared with those who were treated after. A total of 14 patients were identified who were treated following publication of the VA. Differences in stage are presented in Fig. 2. No stage IV patients were identified in the pre-VA group. The mean follow-up period for patients who were given radiotherapy was $16.8 \pm 18$ years (range, $1-57$ ). OS time was
$33.2 \pm 5.2$ and $11.7 \pm 1.7$ years for the pre- and post-VA patients, respectively $(\mathrm{P}=0.413)$ (Fig. 3). In total, 6 patients within each group experienced recurrence. The 2-year disease-free survival rate was $93 \%$ for patients who were treated prior to the VA and $71 \%$ for patients who were treated following the study $(\mathrm{P}=0.001)$. The 2-year laryngectomy-free survival rate was $53 \%$ for pre-VA patients and $78 \%$ for post-VA patients $(\mathrm{P}=0.299)$ (Fig. 4).

Effect of radiotherapy on patients with laryngeal SCC. The mean duration of follow-up in patients treated with radiotherapy was $16.8 \pm 18.5$ years (range, 1-57). In order to examine the effect of radiation on secondary malignant neoplasms (SMNs) in these patients, patients who were treated with radiotherapy and developed a second primary malignancy were compared with patients who did not. Overall, 6 patients developed SMNs, 4 (19\%) received radiotherapy, while the other two (25\%) had no radiotherapy. The OS time was 26.3 years [95\% confidence interval (CI), 9.9-42.6] for patients with SMNs compared with 34.2 (95\% CI, 23.3-45.1) for other irradiated patients $(\mathrm{P}=0.241)$. When assessing the impact of radiotherapy on patients with SMNs, no difference was observed in OS time between those who had a history of radiotherapy and those who did not $(\mathrm{P}=0.441)$.

\section{Discussion}

The present study describes the patient and tumor characteristics, disease history, and treatment outcome of patients $<40$ years of age with laryngeal cancer. The results demonstrated non-significantly higher rates of laryngectomy-free survival in patients who were treated post-VA, with no difference in OS time compared with patients who were treated pre-VA, suggesting that the organ preservation concept should be applied.

Laryngeal cancer in patients younger than 40 years of age is rare. Classical risk factors, including smoking and alcohol, are not as prevalent among young patients as in older ones $(4,5)$. This has led to the assumption that the carcinogenic effect of tobacco is not the primary cause of cancer in young patients and that this disease has a different etiology. Human papillomavirus (HPV) for example, is common in youngsters and is linked to oropharyngeal SCC (11). However, a link between laryngeal cancer and HPV infection remains controversial (6-8). Another possible etiology is diet. Levi et al (12) reported a higher overall recurrence rate of laryngeal cancer in young patients who ate processed meat, although processed meat may represent a more general indicator of an unfavorable diet for laryngeal cancer risk. The present cohort demonstrated lower smoking rates than older patients, but other possible etiologies for the disease could not be investigated due to the retrospective nature of the work.

A number of previous studies compared younger and older patients with laryngeal SCC and did not observe any differences in tumor location or disease stage $(4,13)$. The tumor locations reported in the current study were similar to those previously reported in the literature, with the glottis being the most prevalent location followed by the supraglottis (14). In a longitudinal study of patients with laryngeal cancer, Hoffman et al (15) was unable to define tumor 
Table I. Demographic and clinical characteristics of 29 patients with laryngeal squamous cell carcinoma.

\begin{tabular}{lccc}
\hline Characteristic & $\begin{array}{c}\text { Overall cohort } \\
(\mathrm{n}=29)\end{array}$ & $\begin{array}{c}\text { Pre-VA } \\
(\mathrm{n}=15)\end{array}$ & $\begin{array}{c}\text { Post-VA } \\
(\mathrm{n}=14)\end{array}$ \\
\hline Male: female ratio & $2.6: 1$ & $12: 3$ & $9: 5$ \\
Age, mean \pm SD & $35 \pm 5$ & $34.7 \pm 6$ & $35.1 \pm 3.9$ \\
Smoking (pack/year), & $17(59)$ & $12(80)$ & $5(36)$ \\
$\mathrm{n}(\%)$ & & & \\
Tumor site, n (\%) & & & \\
Glottis & $20(69)$ & $13(86)$ & $7(50)$ \\
Supraglottis & $6(21)$ & $1(7)$ & $5(36)$ \\
$\quad$ Unknown & $3(10)$ & $1(7)$ & $2(14)$ \\
Stage, n (\%) & & & \\
I & $12(41)$ & $8(53)$ & $3(21)$ \\
II & $4(14)$ & $4(27)$ & $1(7)$ \\
III & $8(28)$ & $3(20)$ & $5(36)$ \\
IV & $5(17)$ & $0(0)$ & $5(36)$ \\
Treatment, n (\%) & & & \\
Surgery & $11(38)$ & $7(47)$ & $4(29)$ \\
Radiation & $21(72)$ & $10(67)$ & $11(79)$ \\
Chemotherapy & $5(17)$ & $0(0)$ & $5(36)$ \\
Recurrence, n (\%) & $12(41)$ & $6(40)$ & $6(43)$ \\
Mortality, n (\%) & $15(51)$ & $11(73)$ & $4(29)$ \\
\hline
\end{tabular}

VA, The Veterans Affairs laryngeal cancer study; SD, standard deviation.

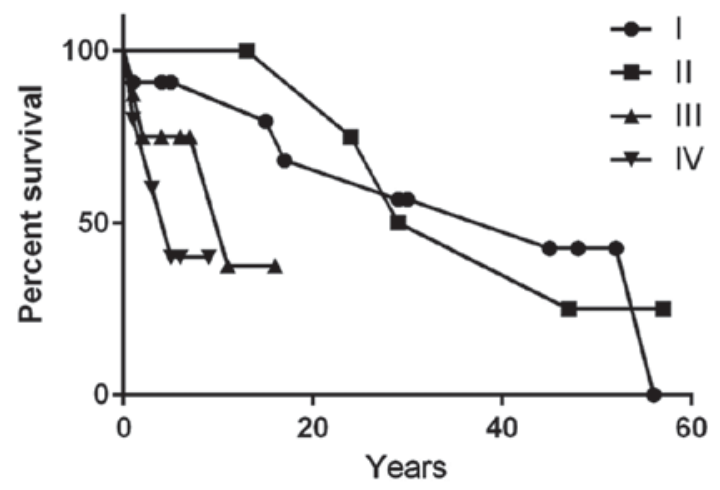

Figure 1. OS time of patients according to their disease stage. Stage IV and III patients had an OS time of $5.4+/-1.4$ and $10.5+/-2.2$ respectively, a significantly worse outcome than patients with earlier stages ( $\mathrm{P}=0.043)$. OS, overall survival.

location in $14 \%$ of patients, similar to the $10 \%$ in the present study. In another study, no difference was reported in histological grade among young patients, and the most common grade was well-to-moderately-differentiated SCC, which is consistent with the current study (16). Several previous studies have addressed survival difference in young patients. A number of these report that youngsters have a poorer prognosis than older patients (16), while others report the survival is the same $(17,18)$ or even better $(4,19-21)$. However,

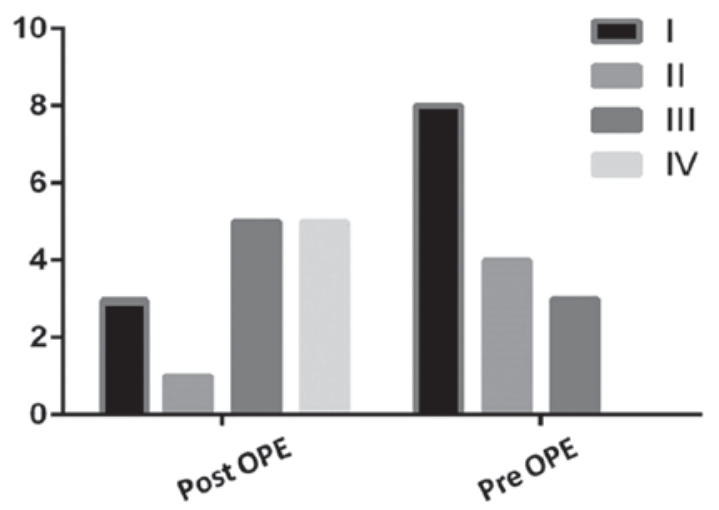

Figure 2. Differences in disease stage in patients treated prior to and following the OPE. No stage IV patients were identified within the pre-OPE group. OPE, organ preservation era.

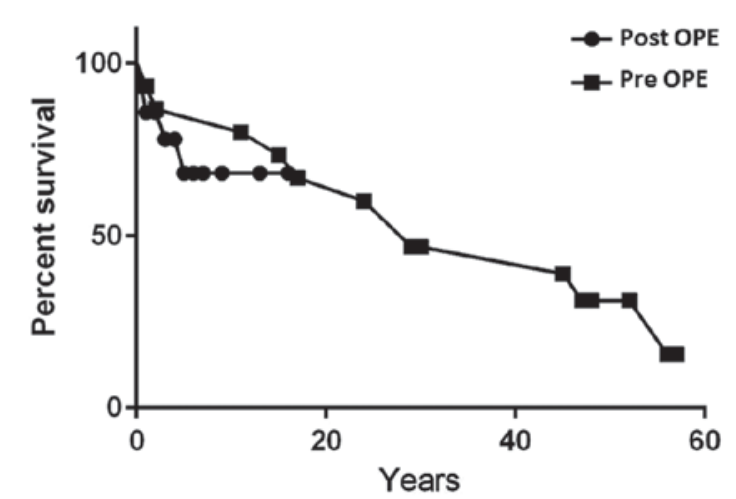

Figure 3. OS time of patients of all stages of disease, pre- and post-OPE. OS was $33.2+/-5.2$ and $11.7+/-1.7$ for pre- and post-OPE, respectively $(\mathrm{P}=0.413)$. OS, overall survival; OPE, organ preservation era.

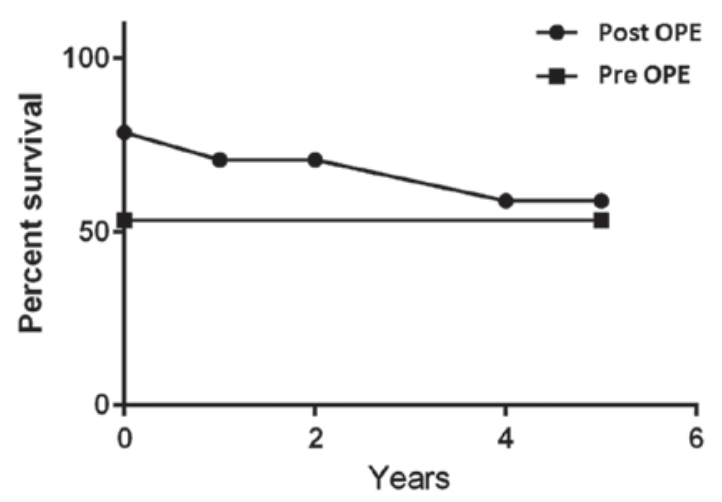

Figure 4. Laryngectomy-free survival of patients prior to and following the OPE. The 2-year laryngectomy-free survival was 78 and $53.3 \%$ for post- and pre-OPE, respectively $(\mathrm{P}=0.299)$.

confounders, including poorer baseline medical status and other comorbidities, most likely serve a role in these observations. Reizenstein et al (18) reviewed 945 patients with laryngeal cancer. Patients $<40$ years of age were treated in a similar manner as older patients, and $77 \%$ were given curative radiotherapy. Similar OS, disease-specific survival and relapse-free survival rates were reported except in patients $>80$ years of age, who did worse in all these parameters (18). 
Overall, SCC in young patients appears to behave in a manner similar to the disease in its traditional population.

The VA and the RTOG-91 (22) changed the treatment dogma towards organ preservation treatment, including chemotherapy and radiotherapy. Today the larynx-preservation approach is a standard, appropriate treatment option even in advanced disease stage (23). There are compelling studies regarding the effect of this shift on survival in laryngeal cancer. For example, Hoffman et al (15) reported of decreased survival in patients with laryngeal cancer, which was attributed to the shift in treatment towards chemoradiotherapy. Despite this, organ preservation treatment is still recommended in advanced stage laryngeal cancer $(23,24)$. A recommendation for laryngectomy in young patients is not given easily. Lee-Preston et al (25) reported a poorer quality of life in young patients treated with laryngectomy and radiotherapy compared with radiotherapy alone, and concluded that younger patients are more likely to require intensive support throughout the treatment period, which should be taken into consideration when discussing treatment options with younger patients. The results of the current study demonstrated a non-significantly higher rate of laryngectomy-free survival and a higher recurrence rate in patients treated following publication of the VA. However, no change in OS time was observed between the two groups despite patients in the post-VA group having more advanced disease stage. Similar OS time and laryngectomy-free survival were demonstrated between the groups even without excluding stage IV patients.

In the past, radiotherapy was avoided in young patients due to the fear of SMNs. Development of SMNs has a negative impact on survival in cases of laryngeal cancer $(26,27)$. The present study demonstrated a trend towards decreased survival in these patients, however, the majority of irradiated patients did not develop SMNs and radiotherapy improved survival in these patients. Furthermore, the results of the current study did not demonstrate a difference in OS time between patients who had SMNs and prior radiotherapy treatment and patients who did not. Albright et al (28) reviewed the Surveillance, Epidemiolosgy and End Results database and identified that patients $<40$ years of age with invasive SCC of the larynx were significantly less likely to develop a second malignancy than older patients. Thus, it may be reasonable to consider radiotherapy in the treatment of young patients.

The present study has certain limitations. Firstly, the small cohort makes it difficult to reach statistical significance. However, one of the aims of the study was to evaluate trends in survival of young patients with SCC of the larynx, similar to Hoffman et al (15), who reported of decreased survival in the organ preservation era. Secondly, the long follow-up time of the study, which spreads over 53 years, may lead to certain imprecision. Nevertheless, treatment modality, date of diagnosis and mortality should not be affected by this possibility.

In conclusion, SCC of the larynx in young adults has the same characteristics and behavior as in older patients. Higher rates of laryngectomy-free survival were noted in patients treated following publication of the VA with no significant difference in survival compared with patients who were treated prior to the VA, suggesting that the organ preservation era did not affect survival.

\section{References}

1. Siegel RL, Miller KD and Jemal A: Cancer statistics, 2016. CA Cancer J Clin 66: 7-30, 2016.

2. Muscat JE and Wynder EL: Tobacco, alcohol, asbestos, and occupational risk factors for laryngeal cancer. Cancer 69: 2244-2251, 1992.

3. Menvielle G, Luce D, Goldberg P, Bugel I and Leclerc A: Smoking, alcohol drinking and cancer risk for various sites of the larynx and hypopharynx. A case-control study in France. Eur J Cancer Prev 13: 165-172, 2004.

4. Singh B, Alfonso A, Sabin S, Poluri A, Shaha AR, Sundaram K and Lucente FE: Outcome differences in younger and older patients with laryngeal cancer: A retrospective case-control study. Am J Otolaryngol 21: 92-97, 2000.

5. Shvero J, Hadar T, Segal K, Abraham A and Sidi J: Laryngeal carcinoma in patients 40 years of age and younger. Cancer 60 : 3092-3095, 1987

6. Li X, Gao L, Li H, Gao J, Yang Y, Zhou F, Gao C, Li M and Jin Q: Human papillomavirus infection and laryngeal cancer risk: A systematic review and meta-analysis. J Infect Dis 207: 479-488, 2013

7. Koskinen WJ, Brøndbo K, Mellin Dahlstrand $\mathrm{H}$ Luostarinen T, Hakulinen T, Leivo I, Molijn A, Quint WG, Røysland T, Munck-Wikland E, et al: Alcohol, smoking and human papillomavirus in laryngeal carcinoma: A Nordic prospective multicenter study. J Cancer Res Clin Oncol 133: 673-678, 2007.

8. Baumann JL, Cohen S, Evjen AN, Law JH, Vadivelu S, Attia A, Schindler JS, Chung CH, Wirth PS, Meijer CJ, et al: Human papillomavirus in early laryngeal carcinoma. Laryngoscope 119: 1531-1537, 2009.

9. Coca-Pelaz A, Rodrigo JP, Takes RP, Silver CE, Paccagnella D, Rinaldo A, Hinni ML and Ferlito A: Relationship between reflux and laryngeal cancer. Head Neck 35: 1814-1818, 2013.

10. No authors listed. Induction chemotherapy plus radiation compared with surgery plus radiation in patients with advanced laryngeal cancer. The department of veterans affairs laryngeal cancer study group. N Engl J Med 324: 1685-1690, 1991.

11. Herrero R, Castellsagué X, Pawlita M, Lissowska J, Kee F, Balaram P, Rajkumar T, Sridhar H, Rose B, Pintos J, et al: Human papillomavirus and oral cancer: The International Agency for Research on Cancer multicenter study. J Natl Cancer Inst 95: 1772-1783, 2003.

12. Levi F, Pasche C, Lucchini F, Bosetti C and La Vecchia C: Processed meat and the risk of selected digestive tract and laryngeal neoplasms in Switzerland. Ann Oncol 15: 346-349, 2004 .

13. Luna-Ortiz K, Villavicencio-Valencia V, Pasche P, Lavin-Lozano A and Herrera-Gómeza Á: Laryngeal cancer in patients younger vs older than 40 years old: A matched-paired analysis. Acta Otorrinolaringologica Esp 62: 113-118, 2011 (In Spanish).

14. van Dijk BA, Karim-Kos HE, Coebergh JW, Marres HA and de Vries E: Progress against laryngeal cancer in The Netherlands between 1989 and 2010. Int J Cancer 134: 674-681, 2014.

15. Hoffman HT, Porter K, Karnell LH, Cooper JS, Weber RS, Langer CJ, Ang KK, Gay G, Stewart A and Robinson RA: Laryngeal cancer in the United States: Changes in demographics, patterns of care and survival. Laryngoscope 116: (9 Pt 2 Suppl 111) 1-13, 2006.

16. Lipkin A, Miller RH and Woodson GE: Squamous cell carcinoma of the oral cavity, pharynx, and larynx in young adults. Laryngoscope 95: 790-793, 1985.

17. Verschuur HP, Irish JC, O'Sullivan B, Goh C, Gullane PJ and Pintilie M: A matched control study of treatment outcome in young patients with squamous cell carcinoma of the head and neck. Laryngoscope 109: 249-258, 1999.

18. Reizenstein JA, Bergström SN, Holmberg L, Linder A, Ekman S, Blomquist E, Lödén B, Holmqvist M, Hellström K, Nilsson CO, et al: Impact of age at diagnosis on prognosis and treatment in laryngeal cancer. Head Neck; 32: 1062-1068, 2010.

19. Rutt AL, Hawkshaw MJ and Sataloff RT: Laryngeal cancer in patients younger than 30 years: A review of 99 cases. Ear Nose Throat J 89: 189-192, 2010.

20. Petrović Z , Stojcić G and Jesić S: Laryngeal carcinoma in younger patients. J Laryngol Otol 110: 934-936, 1996. 
21. Misono S, Marmor S, Yueh B and Virnig BA: Treatment and survival in 10,429 patients with localized laryngeal cancer: A population-based analysis. Cancer 120: 1810-1817, 2014.

22. Forastiere AA, Goepfert H, Maor M, Pajak TF, Weber R, Morrison W, Glisson B, Trotti A, Ridge JA, Chao C, et al: Concurrent chemotherapy and radiotherapy for organ preservation in advanced laryngeal cancer. N Engl J Med 349: 2091-2097, 2003.

23. American Society of Clinical Oncology, Pfister DG, Laurie SA, Weinstein GS, Mendenhall WM, Adelstein DJ, Ang KK, Clayman GL, Fisher SG, Forastiere AA, et al: American Society of Clinical Oncology clinical practice guideline for the use of larynx-preservation strategies in the treatment of laryngeal cancer. J Clin Oncol 24: 3693-3704, 2006.

24. Forastiere AA: Larynx preservation and survival trends: Should there be concern? Head Neck 32: 14-17, 2010
25. Lee-Preston V, Steen IN, Dear A, Kelly CG, Welch AR, Meikle D, Stafford FW and Wilson JA: Optimizing the assessment of quality of life after laryngeal cancer treatment. J Laryngol Otol 118: 432-438, 2004.

26. Fujita M, Rudoltz M, Canady DJ, Patel P, Machtay M, Pittard MQ, Mohiuddin M and Regine WF: Second malignant neoplasia in patients with $\mathrm{T} 1$ glottic cancer treated with radiation. Laryngoscope 108: 1853-1855, 1998.

27. Holland JM, Arsanjani A, Liem BJ, Hoffelt SC, Cohen JI and Stevens KR Jr: Second malignancies in early stage laryngeal carcinoma patients treated with radiotherapy. J Laryngol Otol 116: 190-193, 2002.

28. Albright JT, Karpati R, Topham AK, Spiegel JR and Sataloff RT: Second malignant neoplasms in patients under 40 years of age with laryngeal cancer. Laryngoscope 111: 563-567, 2001. 